\title{
Study for Solving the Path on the Three-dimensional Surface Based on Cellular Automata Method
}

\author{
Yuanzhi Wang \\ Geographic Department, Dezhou University, Dezhou 253023, China \\ Tel: 86-534-898-5841 E-mail:wangyuanzhi1@sina.com.cn
}

The research is financed by Shandong province office of education.No.S07WZ35

\begin{abstract}
For the path optimization problem on the irregular surface of three-dimension, three-dimensional surface is discretized with grid. Based on the parallel character of cellular automata in the cellular space, with the dynamic cellular neighbors, the time evolution interval is defined as the minimum remaining weight. The new shortest path algorithm is structured, based on cellular automaton model. That is to say, through the simple rules of evolution of cellular state, the shortest path is got. The method can achieve the efficiency of ant colony algorithm, and a new way of application of the Cellular Automata model is provided.
\end{abstract}

Keywords: Cellular Automata, The path on three-dimensional surface, Path optimization

\section{The background of the problem}

On the Plane, a straight line is the shortest distance between two points, but for mountain such complex three-dimensional surface, the shortest walking path between the starting point and destination can not be a simple straight line. It is a more complex problem to find the shortest path on the complex three-dimensional surface. In the actual case, such as outdoor sports-climbing, the path of choice depends on human experience. Such an approach has a considerable arbitrariness, and it is hard to find the best route. When the digital map of the complex region is known, and a series of the coordinates and elevation data of discrete points is known, theoretically optimal path can be obtained through a certain algorithm. For instance, Wuhan University, Dr. Ge Xiao San finds the optimal path of the three-dimensional surface through Ant Colony Algorithm. In this study, the way solving the shortest path of a complex three-dimensional surface with cellular automaton algorithm is discussed

\section{Concept of graph}

In mathematics and computer science, graph theory is the study of graphs: mathematical structures used to model pairwise relations between objects from a certain collection. A "graph" in this context refers to a collection of vertices or nodes and a collection of edges that connect pairs of vertices. A graph may be undirected, meaning that there is no distinction between the two vertices associated with each edge, or its edges may be directed from one vertex to another.

In graph theory, the shortest path problem is the problem of finding a path between two vertices (or nodes), and the sum of the weights of its constituent edges is minimized. An example is finding the quickest way to get from one location to another on a road map; in this case, the vertices represent locations and the edges represent segments of road and are weighted by the time needed to travel that segment. The most important algorithms for solving this problem are: Dijkstra's algorithm solves the single-pair, single-source, and single-destination shortest path problems. Bellman-Ford algorithm solves single source problem if edge weights may be negative. A* (Special meaning symbols) search algorithm solves for single pair shortest path using heuristics to try to speed up the search. Floyd-Warshall algorithm solves all pairs shortest paths. Johnson's algorithm solves all pairs shortest paths, and may be faster than Floyd-Warshall on sparse graphs. Perturbation theory finds (at worst) the locally shortest path.

\section{Cellular Automata}

A cellular automaton (CA) is a discrete model studied in computability theory, mathematics, physics, theoretical biology and microstructure modeling. It consists of a regular grid of cells, each in one of a finite number of states, such as "On" and "Off". The grid can be in any finite number of dimensions. For each cell, a set of cells is called its neighborhood (usually including the cell itself) is defined relative to the specified cell. An initial state (time $\mathrm{t}=0$ ) is selected by assigning a state for each cell. A new generation is created (advancing $\mathrm{t}$ by 1 ), according to 
some fixed rule (generally, a mathematical function) that determines the new state of each cell in terms of the current state of the cell and the states of the cells in its neighborhood.

A general cellular automaton is a four-tuple: $A=\left(L_{d}, S, N, f\right)$, Where $L_{d}$ represents d-dimensional cellular space; $\mathrm{S}$ is a discrete and limited set of cellular status; $\mathrm{N}$ represents a collection of all the neighborhood (including the Center Cellular), which is space vector that contains $\mathrm{n}$ different cellular state.

SWolfram have studied one-dimensional cellular automaton rule systems systematically. SWolfram proposed Wolfram rules of one-dimensional cellular automaton in the early 20th century, 80 . However, the model is difficult to handle the discrete cellular space and time for the shortest path graph search, therefore, the following extended cellular automaton model is considered. In the extended cellular automaton model all the vertices will be defined as a cellular set; the vertices Linked with the vertices is defined as the cellular neighbors; the interval of time evolution is defined as the smallest remaining weight changing with the evolution of progress. Hypothetically, a cellular starts from a starting point to its adjacent points on the copy, while the adjacent point of cellular replicates concurrently to the respective adjacent node with the cellular state evolution. Path recorded by the cellular copying to the destination node firstly shall be the shortest path. Cellular only comply with the four states: breed, growth, no load, mature. The solution of the shortest path is proposed based on the above idea.

\section{Seeking the shortest path principle based on CA algorithm}

Cellular automata $A=(L, S, N, f)$ is constructed on n vertex weighted graph $\mathrm{G}(\mathrm{V}, \mathrm{E})$, where, cellular space is

$$
L=\left\{v_{1}, v_{2}, \ldots, v_{n}\right\} ;
$$

neighbor of center cellular is $N\left(v_{x}\right)$; state set is

$$
\mathrm{S}=\left(\mathrm{S} \_\mathrm{N}, \mathrm{S} \_\mathrm{G}, \mathrm{S} \_\mathrm{B}, \mathrm{S} \_\mathrm{M}\right)
$$

where, $\mathrm{S} \_\mathrm{N}$ means that the vertex is in initial state, and is not searched; $\mathrm{S}$ B $\mathrm{B}$ indicates that the vertex is in breeding condition, that is the vertex information will be expanded to neighbor vertex; S_G indicates that the vertex is in the growth state, that is the road is being searched; S_M indicates that the vertex is in mature state, that is the road has been searched.

As shown in Figure 1, evolution rule is $\mathrm{f}$; the current phase is $\mathrm{t}$; the Center cellular is $v_{0}$; the rest weight is $R_{i}\left(v_{0}\right)$. The center cellular state are the following four:

(1) S_M state indicates that the cellular is in mature state, and has been seeking road. Changes need not being made.

(2) S_B state indicates that this vertex has been searching way. The state is to $S \_M$ at the next time.

(3) $\mathrm{S} \_\mathrm{N}$ state indicates that the cellular is in on load state. With examination of the previous neighbor cellular states, if there is any a neighbor (j) of S_B state, the center cellular (i) is to S_G and modify remaining weight of the centre cellular:

$$
R_{i}=\operatorname{dis} \tan c e(i, j), \quad i, j \in L_{d},
$$

where dis $\tan c e(i, j)$ denotes the distance of cellular i to cellular $\mathrm{j}$.

(4) S_ G state indicates that the cellular is in growth state. Previous neighbor cellular state is checked. The minimum residual weight of cellular space is defined as $m r$, and

$$
R_{i}=R_{i}-m r .
$$

If $R_{i}=0$, the center cellular(i) converts to the state of $\mathrm{S} \_\mathrm{B}$ in the next time. Otherwise, if there is any a neighbor cellular on S_B state and

$R_{i}$ updates to

$$
\text { distance }(i, j)<R_{i},
$$

$$
R_{i}=\operatorname{dis} \tan c e(i, j), i, j \in L_{d} .
$$

At the same time, minimum residual weight of the next time changes from $m r^{\prime}$ to

$$
m r^{\prime}=\min \left\{R_{i}, i \in L_{d}\right\} .
$$


There is a specific instance shown in Figure 2.

\section{The concrete solution of the shortest path on three-dimensional surface}

(1) Three-dimensional position data of the study area is imported into, including the plane coordinates and elevation values of the starting point $\mathrm{A}$ and end point $\mathrm{B}$. The problem is seeking the shortest path between $\mathrm{A}$ point and $\mathrm{B}$ point. The distance between $\mathrm{A}$ and $\mathrm{B}$ point will be divided into $\mathrm{n}$ equal portions, and the vertical profile is made over each equal points, so that each vertical profile intersects three-dimensional surface, $n$ profile curves are derived. Then each profile curve are equally to be $\mathrm{m}$ points according to horizontal distance, so that the path search problem of three-dimensional surface is transformed into a discrete optimization problem through gridding. Values of $\mathrm{m}, \mathrm{n}$ are based on the required precision. According to the data source, the appropriate interpolation methods are chosen, so the elevation data for each point of square grids are derived. Profile curve's projection on level segment for three-dimensional surface is called pcp3D for short, and average part curve segment is called apc for short(see Figure 3).

(2) There are cliffs, deep-water zones which can not be through in the complex terrain area, and these regions are known as the three-dimensional obstacles on the surface. For the three-dimensional obstacles on the surface, treatment is the following: for points $C_{i}^{k}(i \in 1,2, \ldots m)$ on the profile curve $P^{k}(k \in 1,2, \ldots n)$, an allow list allowed $_{i}$ is introduced, and allowed ${ }_{i}$ is the set of points on the curve $P^{k+1}$. By calculating the connection between every point on the profile curve $P^{k+1}$ and $C_{i}^{k}$, if line segments intersect any barrier region, the point can not be added to allowed . Otherwise, the point is added to allowed $_{i}$. In accordance with this law, all points that can connect to $C_{i}^{k}$ constitutes allowed $_{i}$.

(3) With $\mathrm{C}++$ language development tools and cellular automaton algorithm, the optimal path is achieved. The main data structure is as follows:

struct points list \{

double $\mathrm{x}$;

double $\mathrm{y}$;

double $\mathrm{z}$;

\}; This data structure describes the three-dimensional coordinates of points.

struct CA_list \{

long * NeighborInformation;

long * BelongCellular;

long * IncludeCellular;

\}; This data structure describes the cellular automata, in which NeighborInformation, Belongcellular and IncludeCellular three fields record the cellular properties.

With Matlab tools, three-dimensional graphics of calculation results are shown in Figure 4 . The shortest path is a tortuous line, winding angle is ninety degrees, which is due to the search for the optimal path is based on the discrete projection grid. In fact, person can not walk such way, so the shortest path is a theoretical inference that is the actual reference of deployment.

\section{References}

BV Cherkassky, AV Goldberg, T Radzik. (1996).Shortest paths algorithms: theory and experimental evaluation, Mathematical programming.

GE Xiaosan, BIAN Fuling. (2007). On Algorithm for 3D Surface Route Optimization Based on Ant Colony Optimization. Geomatics and Information Science of Wuhan University, 32 (4):366-368.

LI Min, XUE Huifeng, WU Xiaojun. (2004). The Shortest Path Search Based on Improved Cellular Automata Extend Model. Microcomputer Applications, 5 (24):92-93.

Yue Yang, Gong Jianya. (1999). An Efficient Implementation of Shortest Path Algorithm Based on Dijkstra Algorithm. Journal of Wuhan Technical University of Surveying and Mapping(wtusm), 24 (3) :209-212. 


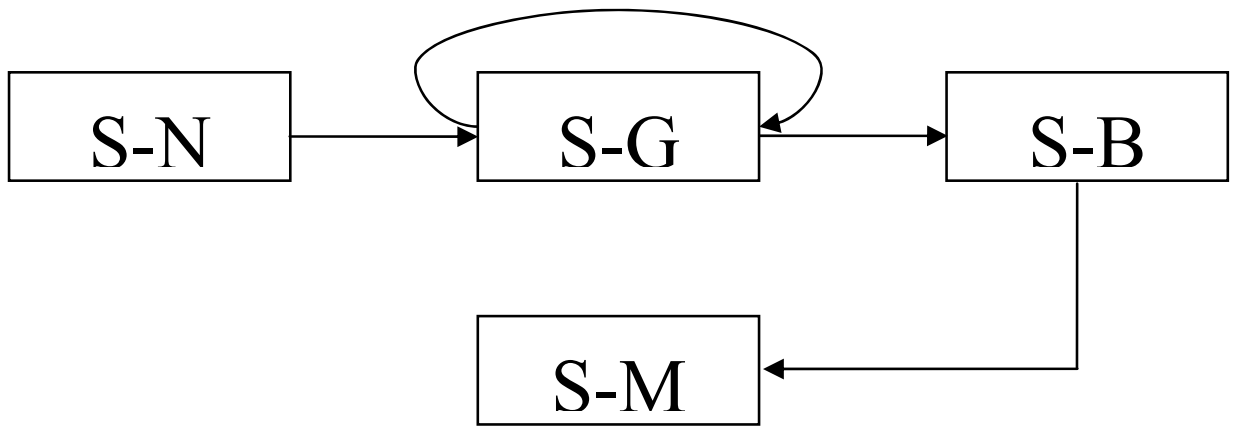

Figure 1. Cellular transformation rules

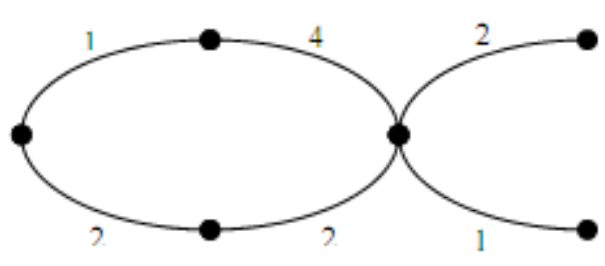

(1)

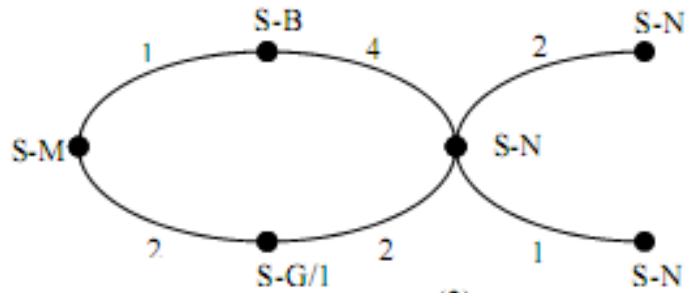

(3)

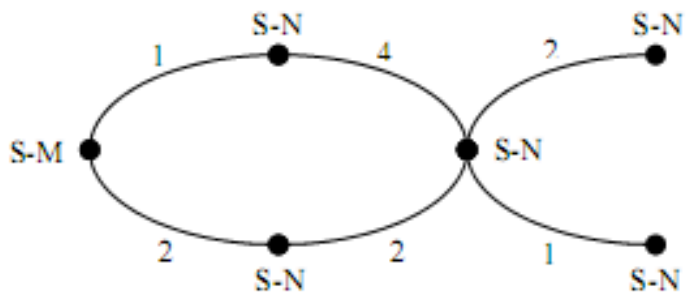

(2)

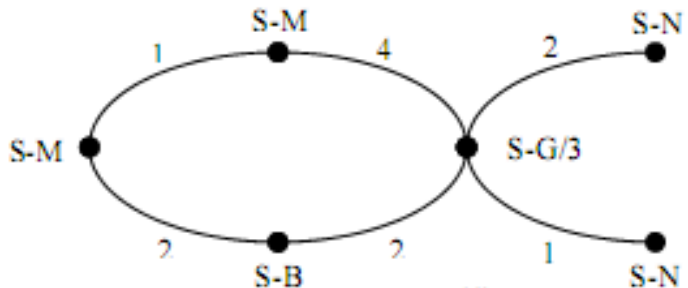

(4)

Figure 2. Examples of cellular conversion process

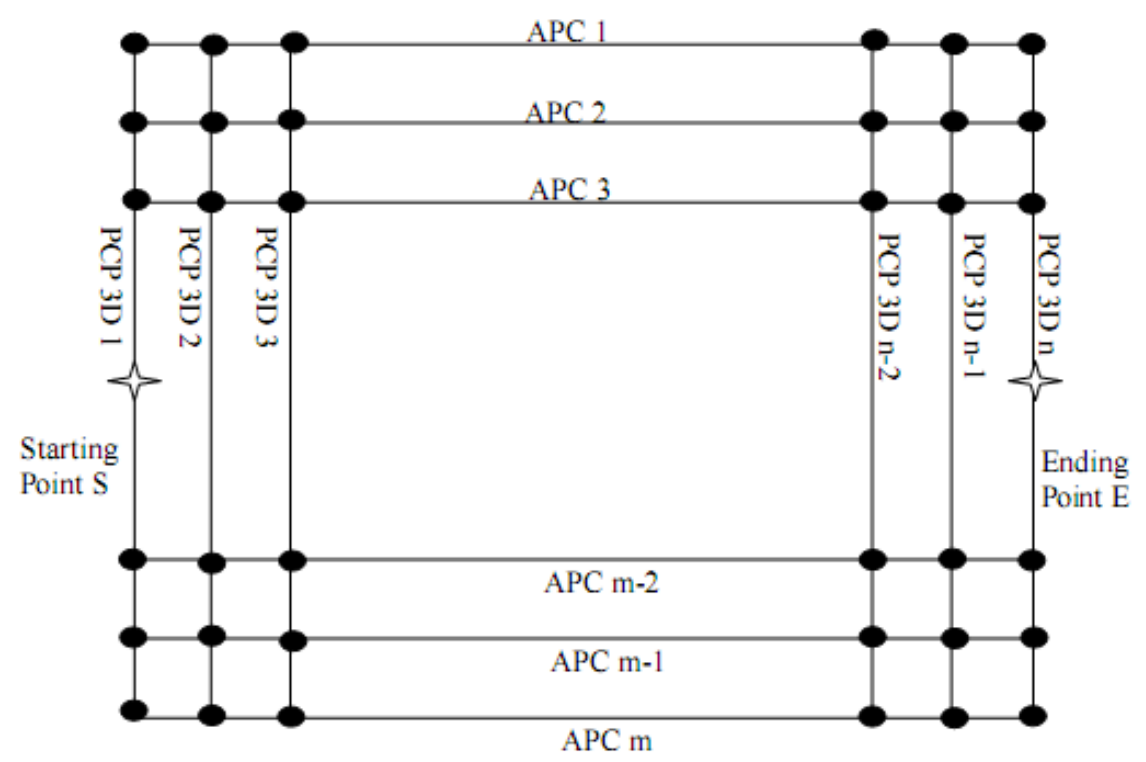

Figure 3. Horizontal projection of three-dimensional surface after discrete gridding 


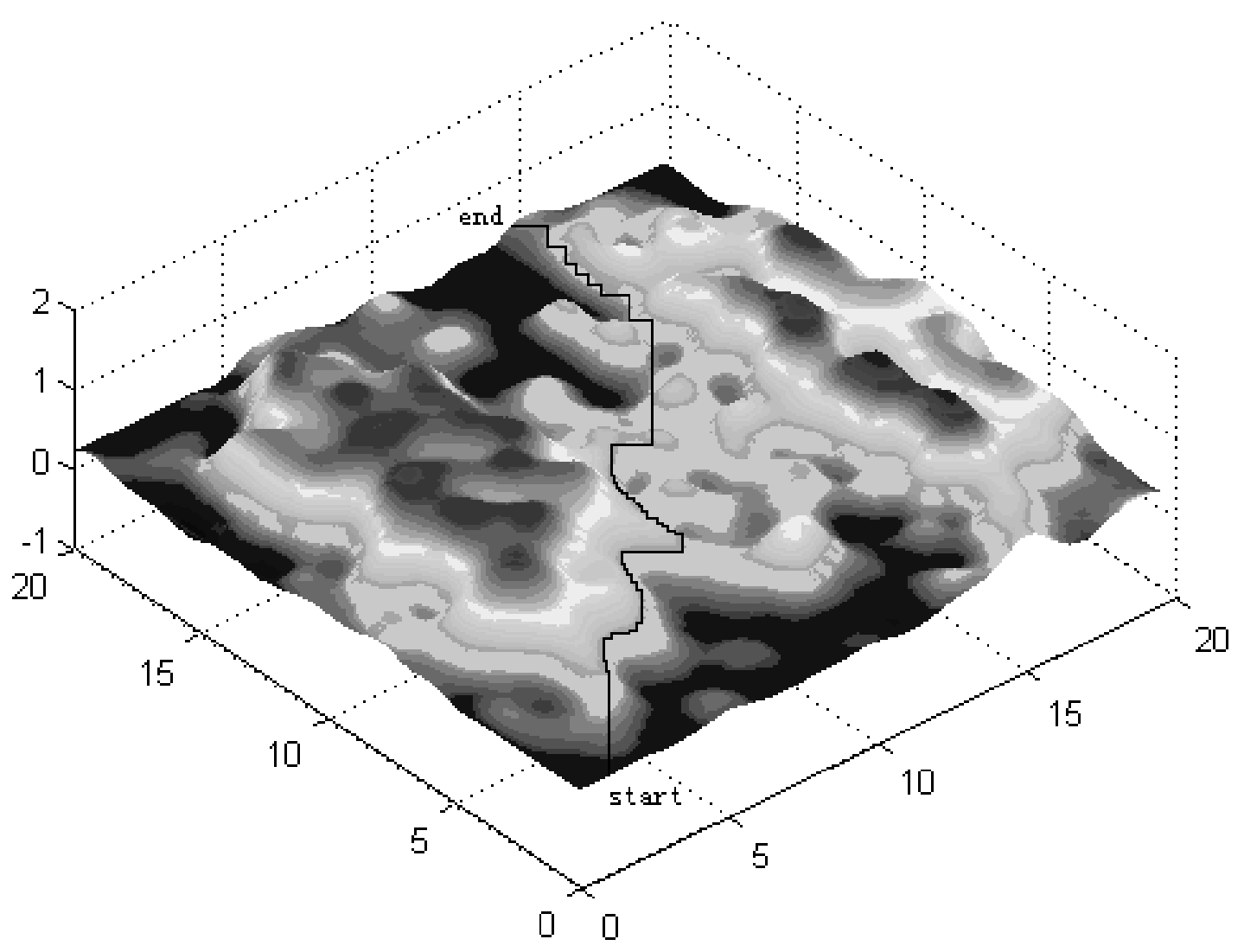

Figure 4. Experimental regional/ cellular automaton optimization results overlay map 